compared to adults control and APC did not reduce this effect. Neutrophil TLR4 expression was significantly increased in response to LPS in NE infants on D3 compared to adults $(p<0.001)$ and has been reduced by APC ( $p=0.03)$. LPS induced monocyte TLR4 was only significantly increased in NE infants D7 $(p<0.001)$. Neutrophil ROI was significantly increased in Adults $(p<0.001)$ and NE infants on D3 ( $p=0.021)$ following LPS and this response were significantly reduced by APC.

Conclusion Neutrophil activation and production of ROI may mediate tissue damage in NE infants. APC modified LPS responses in adults and NE infants on D3 of life. APC may reduce the inflammatory responses secondary to hypoxia and possibly benefit these patients at high risk of inflammatory multiorgan dysfunction.

\section{CARDIAC OUTPUT MEASUREMENTS IN PRETERM NEONATES REQUIRING RESUSCITATION AT BIRTH}

doi:10.1136/archdischild-2012-302724.1098

N Ahmed, V Sundaram, P Kumar. PGIMER, Chandigarh, India

Background The effect of perinatal asphyxia on cardiac output and flow patterns in asphyxiated preterm neonates is less well understood.

Objectives To study the cardiac outputs (left and right ventricleLVO and RVO) and superior vena cava (SVC) blood flow patterns in asphyxiated preterm neonates in first 24 hours of age.

Subject and Interventions Serial echocardiography was done in preterm neonates $<34$ weeks who required resuscitation, at $6 \pm 2$, $12 \pm 2$ and $24 \pm 4$ hours using color Doppler (Sonosite). LVO, RVO and SVC flow velocity were calculated

Results Functional Echo was done in 68 neonates with mean gestation and weight of $31 \pm 1.6$ weeks and $1343 \pm 361 \mathrm{~g}$. Median SVC flow, LVO and RVO at 6, 12 and 24 hrs of age were 109 (70-137), 103 (85-150) and 132 (92-181); 381 (287-493), 421 (337-510) and 408 (324-557); 327 (214-435), 328 (259-467) and 381 (280-501) $\mathrm{ml} / \mathrm{kg} / \mathrm{min}$ respectively. The differences in these three measures between three time points were not statistically significant. A statistically significant increase was seen between SVC flows at 6 versus 24 hours. No difference was observed in these measurements in $21 \%$ vs $100 \%$ oxygen groups.

Conclusions LVO, RVO and SVC flow showed an increasing trend from 6 hrs of age to 24 hrs of age. A significant increase was observed in the SVC flow between 6 and 24 hours of age suggestive of hypoperfusion-reperfusion phenomena. Resuscitating with $21 \%$ or $100 \%$ oxygen did not show any difference in these measurements.

\section{NORMATIVE LEVELS OF INTERLEUKIN 16 IN UMBILICAL CORD BLOOD}

doi:10.1136/archdischild-2012-302724.1099

NM Denihan, AM Looney, GB Boylan, BH Walsh, DM Murray. Neonatal Brain Research Group, Department of Paediatrics and Child Health, Cork University Maternity Hospital, Cork, Ireland

Background and Aims The need for early and accurate prediction of outcome in Hypoxic-Ischemic Encephalopathy (HIE) remains critical. We have previously demonstrated that Interleukin 16 (IL-16) is raised in the umbilical cord blood of infants with moderate and severe HIE and has the potential to be developed as a predictive biomarker. Normal reference ranges for IL-16 in umbilical cord blood have not been previously described. The aim of this study was to determine normative levels of IL-16 in full term neonates using cord blood following uncomplicated deliveries.

Methods Full term infants were recruited as part of an ongoing birth cohort study, the Cork BASELINE Birth Cohort Study. All had cord blood drawn and bio-banked at $-80^{\circ} \mathrm{C}$, within 3 hours of birth. Samples were chosen based on Apgar scores ( $\geq 8$ at $1 \mathrm{~min}, \geq 9$ at $5 \mathrm{~min})$, duration of ruptured membranes $<24 \mathrm{~h}$, temperature in labour $\leq 37^{\circ} \mathrm{C}$, gestational age $\geq 37$ weeks and birthweight centile $\geq 10 \%$. Analysis was performed on plasma EDTA, using ELISA Quantikine ${ }^{\circledR}$ (R\&D Systems, Europe).

Results The study consisted of samples from 48 infants with two different modes of delivery; unassisted vaginal delivery ( $\mathrm{n}=12$ male, $\mathrm{n}=12$ female) and pre-labour elective caesarean section ( $\mathrm{n}=12$ male, $\mathrm{n}=12$ female). The range of all samples was normally distributed between 87.0 and $114.6 \mathrm{pg} / \mathrm{ml}$. Mean (SD) for IL-16 was 103.1 $( \pm 21.9) \mathrm{pg} / \mathrm{ml}$. Levels were not affected by gender or mode of delivery.

Conclusion For the first time we have described the expected range of cord plasma IL-16 levels in healthy term infants.

\section{C-REACTIVE PROTEIN CONCENTRATIONS IN NEONATES WITH HYPOXIC-ISCHAEMIC ENCEPHALOPATHY AND EFFECT OF TOTAL BODY HYPOTHERMIA}

doi:10.1136/archdischild-2012-302724.1100

'S Sanka, 'H Muniraman, ${ }^{2} \mathrm{D}$ Gardner, ${ }^{2} \mathrm{~A}$ Pawaletz, ${ }^{3} \mathrm{C}$ Jennings, ${ }^{3} \mathrm{~A}$ Vayalakkad, ${ }^{3,4} \mathrm{~S}$ Victor, ${ }^{2.5} \mathrm{MA}$ Turner, 'P Clarke. 'Neonatal Unit, Norfolk and Norwich University Hospitals NHS Foundation Trust, Norwich; ' 2 Neonatal Unit, Liverpool Women's Hospital, Liverpool; ${ }^{3}$ Newborn Intensive Care Unit, Central Manchester University Hospitals NHS Foundation Trust; ${ }^{4}$ Developmental Biomedicine Research Group, University of Manchester, Manchester; ${ }^{5}$ Department of Women's and Children's Health, Institute of Translational Medicine, University of Liverpool, Liverpool, UK

Background and Aims Production of C-reactive protein (CRP), an acute phase reactant of hepatic origin, may be affected by perinatal asphyxia. This study tested hypotheses that circulatory CRP concentrations correlate with clinical severity of hypoxic-ischaemic encephalopathy (HIE) and that total body hypothermia modulates CRP response.

Methods Clinical records in three centres were reviewed for neonates $\geq 36$ weeks' gestation admitted between 01/07/06 and $30 / 06 / 11$ with HIE of any severity (grades $1-3$ Sarnat-Sarnat). Participating centres adopted routine cooling at different dates. Data extracted included CRP concentrations in the first postnatal week measured during routine clinical practice, clinical HIE grading, and reception of therapeutic hypothermia. Proportions with raised CRP $(>10 \mathrm{mg} / \mathrm{L})$, and maximum CRP concentrations were assessed according to HIE grade and whether cooled.

Results A raised CRP was present in 150/259(58\%) neonates during the first postnatal week (HIE1: 30/73[41\%], HIE2: 83/129[64\%], HIE3: $37 / 57[65 \%$ ], $\mathrm{p}=0.003$ ) but elevated maximum concentrations (peaking median day 3 ) did not differ between HIE grades (median [range] HIE1: 31.3 [10.0-188.1] mg/L, HIE2: 32.5 [10.0-305.9] mg/L, HIE3: 34.0 [10.2-346.5] mg/L, $\mathrm{p}=0.48)$. A raised CRP was present in $117 / 187(63 \%)$ cooled and $33 / 72(46 \%)$ non-cooled infants ( $p=0.02)$, but their peak CRP concentrations did not differ (median [range] CRP cooled vs. non-cooled: 31.9 [10.0-346.5] mg/L vs. 53.0 [10.4188.1] mg/L, $\mathrm{p}=0.26$ ).

Conclusion A raised CRP is a common finding in the first postnatal week in neonates admitted with HIE and is found in most infants with moderate-severe HIE. Peak CRP concentrations did not differ with clinical HIE grade and whole body hypothermia did not significantly affect peak CRP concentrations.

\section{ARE LACTAT DEHYDROGENASE AN.D NEURON SPECIFIC ENOLASE ANALYSES GOOD DIAGNOSTIC TOOLS FOR ASSESSING EXTENSION OF PERINATAL HYPOXIC- ISCHEMIC BRAIN INJURY?}

doi:10.1136/archdischild-2012-302724.1101

${ }^{1} \mathrm{~B}$ Vasiljevic, ${ }^{2} \mathrm{~S}$ Maglajic, ${ }^{3} \mathrm{M}$ Gojnic, ${ }^{4} \mathrm{D}$ Lutovac, ${ }^{5} \mathrm{D}$ Bogicevic. ${ }^{1}$ Neonatology, Institute of Gynecology and Obstetrics, Clinical Centre of Serbia; ${ }^{2}$ Neonatology, University Children's Hospital; ${ }^{3}$ Perinatology, Institute of Gynecology and Obstetrics, Clinical Centre 
of Serbia; ${ }^{4}$ nstitute of Biochemistry, Clinical Centre of Serbia; ${ }^{5}$ University Children's Hospital, Belgrade, Serbia

Background Diagnosis of perinatal hypoxic-ischemic encephalopathy (HIE) and early prediction neurological outcome is important and difficult. The aim of this study is to determine the value of neuron specific enolase (NSE) and lactate dehydrogenase (LDH) analysis in blood serum (BS) and cerebrospinal fluid (CSF) for evaluating severity HIE and predicting long term outcome in nenates with perinatal asphyxia.

Method 90 neonates (>32 weeks gestation) with perinatal HIE were enrolled prospectively. Perinatal HIE was categorised into three stages according Sarnat and Sarnat clinical scoring system and changes seen on amplitude integrated electroencephalography. NSE and $\mathrm{LDH}$ analysis in BS and CSF were taken during first 48h of age. Neurodevelopment outcome was assessed at 12 months of corrected gestational age using Denver Developmental Screening Test. Results Concentrations of NSE and LDH in CSF were significantly higher in neonates with advanced stage of HIE and corresponded well with subsequent neurodevelopment outcome $(p<0.01)$. Concentrations of $\mathrm{LDH}$ in $\mathrm{BS}$ were significantly higher in neonates with advanced stage of HIE and corresponded well with MODS $(p<0.01)$ and subsequent neurodevelopment outcome $(p<0.01)$ while concentrations of NSE in BP were no significantly higher in neonates with advanced stage of HIE ( $p>0.5)$.

Conclusions NSE and LDH analysis in CSF are accurate diagnostic tool for assessing extension of hypoxic-ischemic brain damage and early identification neonates with perinatal HIE who are at high risk of developmental delay. LDH analysis in BP also might offer an inexpensive, safe and simple prognostic tests for evaluating nenates with perinatal HIE

\section{USE OF SYNTHETIC COLLOIDS COMPARING NORMAL SALINE FOR NEURORESUSCITATION IN TERM NEWBORNS WITH SEVERE HIE}

doi:10.1136/archdischild-2012-302724.1102

D Surkov. NICU, Regional Children's Hospital, Dnipropetrovs'k, Ukraine

Background and Aims There is limited data according the use of $6 \%$ solution of hydroxyethyl starch $130 / 0.42$ in volemic resuscitation for neonates. The aim of the study was to compare the efficiency of $6 \%$ HES 130/0.42 and normal saline in the intensive care of term newborns with severe HIE.

Methods 15 full-term neonates with HIE were included. Score Sarnat II was in 9 newborns and Sarnat III was in 6 babies. The mean gestation age was $39.3 \pm 0.2$ weeks. Apgar score at $1^{\text {st }}$ minute was $3.8 \pm 0.1$ and at $5^{\text {th }}$ minute was $6.0 \pm 0.1$. Routine hemodynamic parameters, lab studies and cerebral perfusion pressure $(\mathrm{CPP}=1.1$ (Vs - Vd)/PI - $5 \mathrm{~mm} \mathrm{Hg}$ ) by transfontanel Doppler (Aaslid R., 1986) were collected at admission, in 1 hour and 3 hours after volume expanders infusion. The volume of normal saline was $20 \mathrm{ml} / \mathrm{kg}$ and of $6 \%$ HES $130 / 0.42$ was $10 \mathrm{ml} / \mathrm{kg}$. Results. Mean blood pressure at the 3 stages of study in response to $6 \%$ HES $130 / 0.42$ administration was 48-55-55 mm Hg, after normal saline 51-53-49 mm Hg. Cerebral perfusion pressure after administrarion of HES 130/0.42 was 7.7-11.8-15.7 mm Hg, after normal saline 14.1-17.6-17.1 $\mathrm{mm} \mathrm{Hg}$. Conclusions Use of HES 130/0.42 results in stable increasing of cerebral perfusion with normalizing of resistance index in front cerebral arteries. The effect occurs after administration of HES and remains up to 3-6 hours. Applying of HES 130/0.42 may be therapy of choice in low cerebral perfusion and/or hemodynamic instability in newborns with severe HIE.

\section{3 \\ NEW POSSIBILITY OF THE USE OF PREPARATION OF} LAZOLVAN AT PREMATURE INFANTS

doi:10.1136/archdischild-2012-302724.1103
MM Chuyko. The National Medical University of the name of Danylo Halytskiy, Lviv Ukraine

Introduction Ambroxol hydrochloride (drug Lazolvan) inherent anti-inflammatory and antioxidant effects that may have an impact onreducing the frequency and severity of intraventricular hemorrhage (IVH).

Purpose Installing the clinical efficacy of intravenous slow (15 min)administration of the drug Lazolvan (single dose $7.5 \mathrm{mg}$ twice a day from the first days of life for 7 days).

Methods In retrospective case-control study included 50 infants with gestational age less than 35 weeks. The main group $(n=18)$ received Lazolvan, control group not received. Before the beginning of the study formed the group did not differ among themselves on basic clinical and demographic indicators. Statistically significant group differ among themselves RDS third degree by the core group of children $-27.8 \%$ vs $6.26 \%$; $=0.03$. Clinical efficacy of the drug Lazolvan determined in terms of relative risk (RR) of IVH an indicator of patient who need to treat (NNT).

Results The relative risk of IVH in the main group 2.7 times less compared with the control group. At 2.17 times more often in terms of relative risk of IVH second degree occurred in the control group children. The treatment of 6.25 premature infants prevents a case of IVH. Newborn core group of perhaps less treated in the intensive care unit, respectively (days) $12.88 \pm 3.07$ vs $9.07 \pm 3.73$, $\mathrm{p}=0.0003$.

Conclusions The positive results from the use of Lazolvan in the complex treatmet of premature infants with risk of IVH prove its clinical effectiveness and extend its use.

\section{LONG-TERM OUTCOME OF TERM NEWBORNS WITH PERINATAL ASPHYXIA - PREDICTIVE FACTORS}

doi:10.1136/archdischild-2012-302724.1104

${ }^{1} \mathrm{~A}$ Avasiloaiei, ${ }^{2} \mathrm{C}$ Dimitriu, ${ }^{3} \mathrm{~A}$ Bivoleanu, 'L Paduraru, ${ }^{1} \mathrm{M}$ Stamatin. 'Neonatology; ${ }^{2}$ Biochemistry, Gr.T. Popa University of Medicine and Pharmacy; ${ }^{3}$ Neonatal Intensive Care Unit, Cuza-Voda Hospital of Obstetrics and Gynaecology, lasi, Romania

Perinatal asphyxia represents the second most important cause of death in the NICU and an important source of neurologic long-time sequelae.

Aim To identify long-term predictive factors for neurologic sequelae in term newborns with perinatal asphyxia.

Material and Methods We conducted a prospective study on 67 term newborns with perinatal asphyxia, admitted to our NICU between 2010-2011. The following parameters were followed: Apgar scores at 1, 5, 10 minutes, cord blood $\mathrm{pH}$, neurologic disorders, creatine-kinase (CK), lactate dehydrogenase ( $\mathrm{LDH})$, total antioxidant status (TAS) at 4, 12, 24, 48, 72 hours and 7 days, follow-up after discharge until 18 months of age.

Results Incidence of perinatal asphyxia in the NICU was $3.76 \%$. Mean Apgar score at 1 minute was 3.58 and at 5 minutes 5.33 , thus indicating the efficiency of resuscitation. Mean blood cord $\mathrm{pH}$ was 7.04. During the first 12 hours of life, all newborns had neurologic disorders. After the first 72 hours, this aspect was only present in $53.7 \%$ of the newborns. TAS was lowest at 12 hours (0.92 mmol/L), not reaching normal values at any moment. Survival was $91.04 \%$ and was correlated with ph $(p=0.012)$, CK $(p=0.04)$, LDH $(p=0.02)$, but not TAS $(p=0.063)$. Neurologic sequelae decreased progressively, reaching $10.45 \%(n=7)$ at 18 months. CK and LDH were predictive for sequelae ( $p=0.01)$, but not the Apgar score or TAS ( $p=0.08$ ).

Conclusion Routine determinations for blood cord $\mathrm{pH}, \mathrm{CK}$ and $\mathrm{LDH}$ can become valuable markers of long-term outcome for newborns with perinatal asphyxia, while TAS remains for further research. 